\title{
Notch-4 contributes to the metastasis of salivary adenoid cystic carcinoma
}

\author{
LIN-CAN DING $^{1 *}$, LIN SHE $^{1 *}$, DA-LI ZHENG ${ }^{2}$, QING-LING HUANG ${ }^{2}$, JIN-FENG WANG $^{1}$, \\ FEI-FEI ZHENG ${ }^{1}$ and YOU-GUANG LU ${ }^{1}$
}

\author{
${ }^{1}$ Department of Preventive Dentistry, Hospital of Stomatology, Fujian Medical University, 246 Yangqiao Zhong Road, Fuzhou \\ 350002; ${ }^{2}$ Molecular Medicine Research Center, Fujian Medical University, 88 Jiaotong Road, Fuzhou 350004, P.R. China
}

Received March 26, 2010; Accepted May 13, 2010

DOI: $10.3892 /$ or_00000868

\begin{abstract}
The Notch signaling pathway is important for cell-cell communication; it is involved in gene regulation mechanisms that control multiple cell differentiation processes during embryonic and adult life. Notch is present in all metazoans, and vertebrates possess four different Notch receptors: Notch-1, Notch-2, Notch-3, and Notch-4. The aim of the present study was to identify the role of Notch protein in the metastasis of salivary adenoid cystic carcinoma (SACC). Real-time PCR results showed that Notch-1, Notch-2, and Notch-4 were upregulated in the highly metastatic SACC cell line ACC-M, compared to ACC-2, a SACC cell line with low metastatic ability. Knockdown of Notch-4 by small interfering RNA efficiently inhibited the invasion of ACC-M cells. Notch-4 expression was significantly higher in the clinical samples with metastasis and recurrence compared to that in control $(\mathrm{p}<0.05)$, shown by immunohistochemistry analysis. These results indicate that Notch-4 may play an important role in SACC metastasis.
\end{abstract}

\section{Introduction}

SACC is one of the most common malignant neoplasms of salivary glands. Lima et al reported that adenoid cystic carcinoma represented the most prevalent malignant neoplasm $(22.41 \%)$ in an analysis of 245 cases (1). SACC occurs

Correspondence to: Dr You-Guang Lu, Department of Preventive Dentistry, Hospital of Stomatology, Fujian Medical University, 246 Yangqiao Zhong Road, Fuzhou 350002, P.R. China E-mail: fjlyg63@163.com

${ }^{*}$ Contributed equally

Abbreviations: SACC, salivary adenoid cystic carcinoma; HLMCL, high and low metastatic cell lines; RFDD-PCR, restriction fragment differential display-PCR; PBS, phosphate-buffered saline; siRNA, small interfering RNA

Key words: oral cancer, salivary adenoid cystic carcinoma, Notch-4, metastasis, RNA interference most frequently in women aged 50-60 years (2). The treatment of SACC is a complicated combination mainly based on surgical resection and local radiation. Most SACC patients survive more than 5 years after surgery and postoperative radiation therapy. However, the 10-year survival rate drops to $40 \%$, due to the locoregional recurrence and distant metastasis often targeting lung and blood vessels. The cumulative metastasis rate at 5 and 10 years of SACC patients was 70 and $100 \%$, respectively (3).

High and low metastasis cell lines (HLMCL) are considered ideal experimental models to investigate the mechanisms of metastasis and invasion in SACC. Lu et al have generated the gene expression profiles of HLMCL of SACC using restriction fragment differential display-PCR (RFDD-PCR), which can screen the related differentially expressed genes more accurately and efficiently (4). By this method, we found that Notch family members are deregulated in highly metastatic SACC cell lines, ACC-M, indicating a potential role of Notch signaling in SACC metastasis. SACC metastasis is a complex process that relates to a number of genes, such as $\beta$ catenin, MMPs, and cyclin D1 $(4,5)$. The Wnt signaling pathway also plays a pivotal role in SACC carcinogenesis and metastasis (5).

Many studies demonstrated that Notch family is involved in the regulation of differentiation, proliferation, and apoptotic processes $(6,7)$. Notch is a transmembrane protein with a modular architecture containing many repeats of a protein module resembling epidermal growth factor (EGF) and three membrane-proximal Lin $12 /$ Notch/Glp-1 (LNG) repeats. The intracellular domain has four distinct regions, the RAM domain, the ankyrin repeats, a transcriptional activator domain (TAD), and the PEST (proline-glutamate-serine-threoninerich) sequence (8). Human Notch receptor family members include Notch-1, Notch-2, Notch-3, and Notch-4. Notch-4 only has one nuclear localization sequence present before the ankyrin repeats, and it lacks the TAD domain. Notch-1 is a tumor suppressor gene, directly regulated by p53 (9). Notch signaling (Notch-4) is related to the deregulation of developmental and differentiation pathways, as well as cell fate misspecification, in the development of oral squamous cell carcinoma. Leethanakul et al demonstrated that Notch signaling may contribute to squamous cell carcinogenesis, and it should be considered to be a candidate marker of this disease (10). Notch proteins play an important role in many 
Table I. Accession number, primer sequence, dissociation curve, and the expression levels of Notch family members in our gene profiles.

\begin{tabular}{lcc}
\hline Accession & \\
none & Primer sequence \\
\hline
\end{tabular}

Notch-1 NM 017617 GGAAGTTGAACGAGCATAGTCC GCATGATGCCTACATTTCAAGA

Notch-2 NM 024408 TATGTTCAAGTGCAGGAATTGG AGAGTCGGGAATTCACCTGTTA

Notch-3 NM 000435 TGAGGGTCAGAATTGTGAAGTG ATAGAGCACTCGTCCACATCCT

Notch-4 NM 004557 CTTCTACTCCGCTTCCTTGCTG' ATCAACTTCTGCCTTTGGCTTC'

ACTB NM 001101 GACAGGATGCAGAAGGAGATCA TTTTAGGATGGCAAGGGACTTC

cancers. However, the effects of Notch proteins on SACC metastasis are poorly understood. In this study, we investigated the role of Notch family members in SACC metastasis.

\section{Materials and methods}

Cell line and tissues. The HLMCL of SACC in our study was sponsored by the Oncology Laboratory of the Department of Stomatology in Shanghai 9th People's Hospital. Tissue samples were screened by the First Affiliated Hospital of Fujian Medical University and Fujian Medical University Union Hospital. Twenty-three cases positive for metastasis and recurrence and 57 cases without metastasis and recurrence were included.

Culture of ACC-M and ACC-2. ACC-M and ACC-2 were cultured in 10-cm plates. Cells were grown in RPMI-1640 (Gibco, USA) supplemented with 15\% heat-inactivated fetal bovine serum (FBS, Gibco) and cultured in a humidified atmosphere of $5 \% \mathrm{CO}_{2}$ at $37^{\circ} \mathrm{C}$. The medium was changed every 2-3 days until the cells reached $75 \%$ confluence, after which the non-adherent cells were washed off with PBS, and the adherent cells were trypsinized with $0.25 \%$ trypsin and $0.01 \%$ EDTA (in a ratio of $1: 1$ ). Then, RPMI-1640 was added to stop the reaction after the cells became rounded. Plasma shrinking and intercellular gap widening were observed using an inverted microscope. The cells were seeded and recultured in the incubator after counting. Cells of passage 4 were prepared for further RNA extraction.

Real-time PCR. Total RNA was extracted from $5 \times 10^{6}$ cells using TRIzol Reagent (Invitrogen, USA). Based on the absorbance at $260 \mathrm{~nm}$, RNA samples were diluted to the same concentration and then, reverse transcribed to cDNA by PrimeScript $^{\mathrm{TM}}$ RT reagent Kit (Takara, Japan). Real-time PCR was performed with SYBR-Green-PCR master mix (Takara). The reaction was carried out in a TP800 (Takara). The primers used for B-actin (ACTB), Notch-1, Notch-2, Notch-3, and Notch-4 are shown in Table I. Each PCR reaction mixture and cycle conditions were carried out according to the SYBRGreen Quantitative PCR Protocol. Melting curve was determined after 40 cycles at $94^{\circ} \mathrm{C} 30 \mathrm{sec}, 94^{\circ} \mathrm{C} 10 \mathrm{sec}$, and $60^{\circ} \mathrm{C}$ $30 \mathrm{sec}$. The fluorescence value of the first 12 cycles was set as the baseline. The housekeeping gene, ACTB, served as the internal control. The $\mathrm{Ct}$ values of all samples were adjusted. Data were analyzed using the method previously reported by Livak and Schmittgen (11).

Immunohistochemistry. For the immunohistochemical assays, $5-\mu \mathrm{m}$ thick tissue sections were mounted on slides coated with poly-L-lysine. After deparaffination in xylene, these sections were rehydrated in a decreasing gradient of ethanol and then, washed for $10 \mathrm{~min}$ in phosphate-buffered saline (PBS) ( $\mathrm{pH} \mathrm{7.2).} \mathrm{The} \mathrm{endogenous} \mathrm{peroxidase} \mathrm{activity} \mathrm{was}$ inhibited by incubation in methanol containing $3 \% \mathrm{H}_{2} \mathrm{O}_{2}$ for $10 \mathrm{~min}$. After several washes in PBS, the sections were blocked with a universal blocking reagent (Maxin, USA) for $10 \mathrm{~min}$ at room temperature. A goat polyclonal Notch-4 (R\&D, USA) primary antibody was applied at a 1:200 (1 $\mu \mathrm{g} \mathrm{IgG/ml)}$ dilution for $1 \mathrm{~h}$ at room temperature. After several washes in PBS, the sections were incubated with a biotin-conjugated secondary antibody (Maxin, USA) for $10 \mathrm{~min}$ at room temperature. After several washes in PBS, the sections were incubated with streptavidin-peroxidase (Maxin) for $10 \mathrm{~min}$ at room temperature. The sections were rinsed with PBS, and the antibody complexes were visualized by incubation with diaminobenzidine tetrahydrochloride (DAB) chromogen (Maxin). The sections were then counterstained with hematoxylin (Dako, DEN), dehydrated, and examined by light microscopy.

RNA interference using small interfering RNA. Two siRNAs against Notch-4 were designed by the Whitehead Institute web server (http://jura.wi.mit.edu/bioc/siRNAext/) and chemically synthesized (Shanghai GenePharma Co., Shanghai, China) for targeting different coding regions of this gene. The sequence information is as follows: siRNA-3790 (5'-CAACGGGCAC UGUGAGAAAdTdT-3' and 5'-UUUCUCACAGUGCCCG UUGdTdG-3') targeting the 3790-3808 nt of SCARA-5 and siRNA-5407 (5'-GGCGGACGUCGCUCACCAAdTdT-3' and 5'-UUGGUGAGCGACGUCCGCCdGdG-3') targeting the 5407-5425 nt of Notch-4. In addition, a negative control, siRNA-NC (5'-UUCUCCGAACGUGUCACGUdTdT-3' and 5'-ACGUGACACGUUCGGAGAAdTdT-3'), was also synthesized. All the above siRNAs were transfected into ACC-M cell lines using Lipofectamine 2000 Transfection Reagent (Invitrogen) according to the manufacturer's instructions.

SDS-PAGE and immunoblot analysis. Briefly, $30 \mu \mathrm{g}$ of protein from each transfection of ACC-M were separated by SDSPAGE using a $6 \%$ gel (for Notch-4) or $12 \%$ gel (for GAPDH), followed by electrophoretic transfer to a PVDF membrane (Amersham Biosciences). The blots were separately incubated with the primary antibodies against Notch-4 (1:100, R\&D, USA) or GAPDH (1:1000, Santa Cruz, USA). The 
A

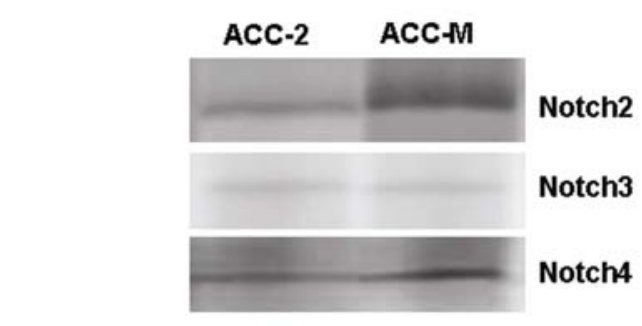

B

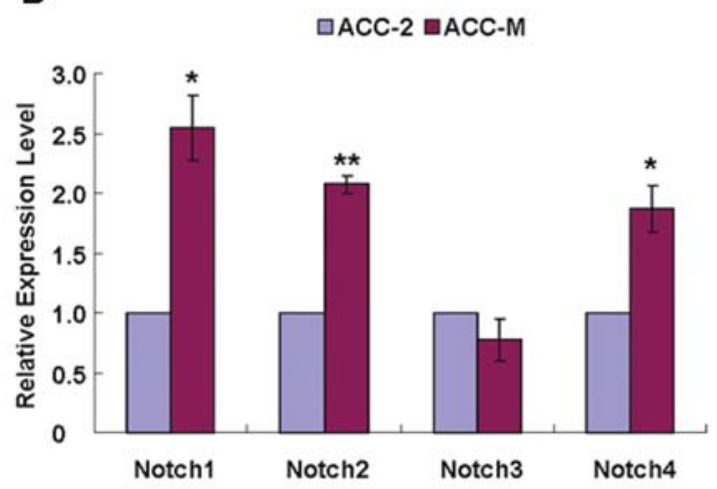

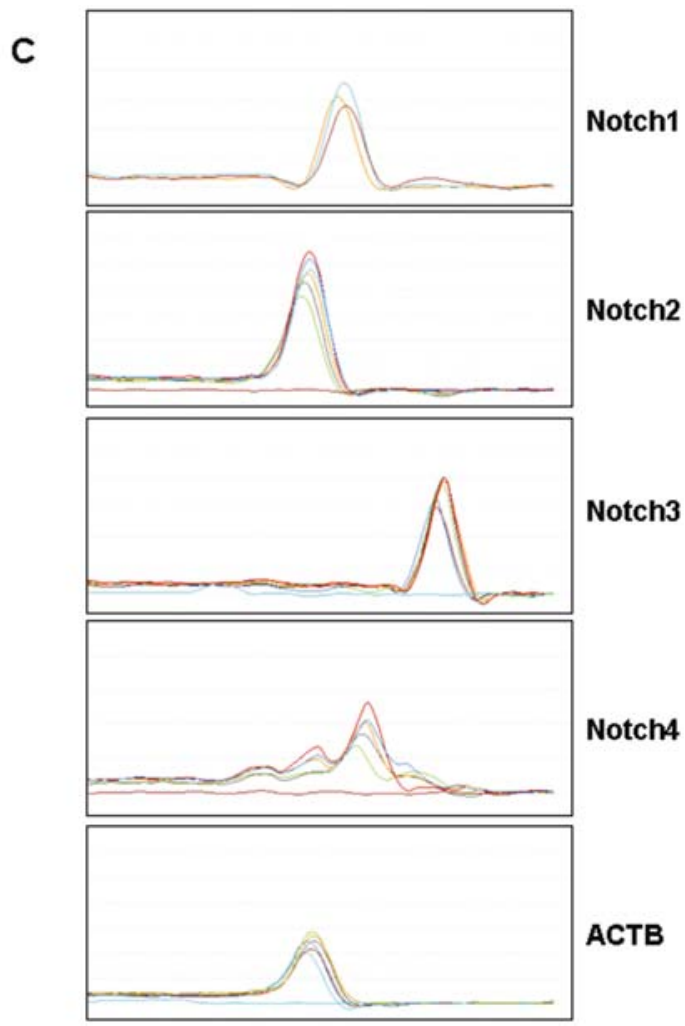

Figure 1. Verification of the expression of Notch family members in ACC-M and ACC-2 cells. (A) The corresponding bands for Notch-2, $-3,-4$ by restriction fragment differential display-PCR. Notch-1 was not listed in this method and Notch-2 and Notch-4 were upregulated in ACC-M cells compared to ACC-2. (B) The results of real-time PCR. The relative expression levels in ACC-M cells were normalized to the values in ACC-2 cells that were set to be 1, as control. (C) The melt chart of each gene as indicated.

primary antibodies were recognized by alkaline phosphataseconjugated secondary antibodies, and the immunoreactive protein bands were visualized using CDP STAR reagents (Roche, IN, USA). The signals were scanned with a densitometer for semi-quantification of the signal intensity.

In vitro cell invasion assay. Cell invasion assays were performed using 24 -well transwell chambers $(8-\mu \mathrm{m}$ pore size, BD Sciences) coated with Matrigel (354480, BD Sciences, USA). ACC-M cells were serum-starved overnight, trypsinized, and washed three times with DMEM containing 1\% FBS. A total of $1 \times 10^{5}$ cells was then suspended in $500 \mu 1$ of DMEM containing $1 \%$ FBS and added to the upper chamber, while $750 \mu \mathrm{l}$ of DMEM containing 10\% FBS and $10 \mu \mathrm{g} / \mathrm{ml}$ fibronectin (356008, BD Sciences) were placed in the lower chamber. For the control, DMEM containing 1\% FBS was added to the lower chamber. After $48 \mathrm{~h}$ of incubation, Matrigel and cells remaining in the upper chamber were removed by cotton swabs. Cells on the lower surface of the membrane were fixed in $4 \%$ paraformaldehyde and stained with $0.5 \%$ crystal violet. Cells in at least six random microscopic fields (at x200) were counted and photographed. All experiments were performed in duplicate and repeated three times.

Statistical analysis. The statistical analysis of Notch-4 immunoreactivity was performed by rank-sum test. The statistical analysis of PCR and in vitro cell invasion assay was performed by Student's t-test. $\mathrm{p}<0.05$ was considered statistically significant.

\section{Results}

Notch family members are upregulated in the highly metastatic cell line $A C C-M$. Notch family members play an important role in a variety of developmental processes by controlling cell fate decisions. In our previous study, we found that Notch family members were deregulated based on the gene expression profiles of the adenoid cystic carcinoma cell lines ACC-M and ACC-2 (4), as shown in Fig. 1A. In this study, real-time PCR was first used to verify the differential expression of all four Notch family genes in ACC-M and ACC-2 cells. As shown in Fig. 1B and C, Notch-1, Notch-2, and Notch-4 were upregulated in the highly metastatic adenoid cystic carcinoma cell line, ACC-M, compared to the low metastatic cell line, ACC-2 $(\mathrm{p}<0.05, \mathrm{n}=3)$, suggesting that these Notch family members may contribute to metastasis of adenoid cystic carcinoma.

Knockdown of Notch-4 inhibits the invasion of ACC-M cells. To investigate the contribution of Notch-1, Notch-2, and Notch-4 to the metastasis of adenoid cystic carcinoma, the small interfering RNA (siRNA)-mediated knockdown of Notch-1, Notch-2, and Notch-4 was employed in ACC-M cells, and cell invasion assays were performed using 24-well transwell chambers. As expected, both siRNAs targeting Notch-4 (siRNA-3790 and siRNA-5407) efficiently reduced Notch-4 expression in ACC-M cells compared to the negative control, siRNA-NC, as shown by Western blot analysis (Fig. 2A), and they significantly inhibited the invasion of 
A

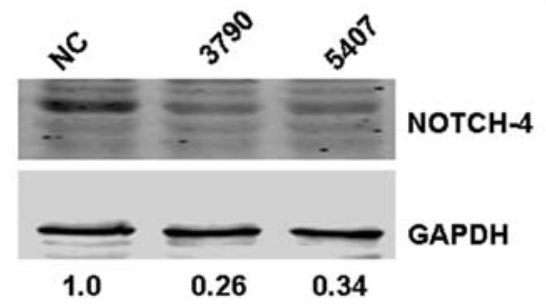

B

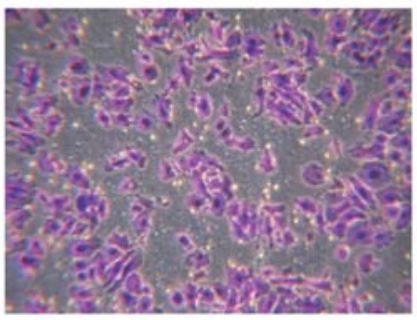

NC
C

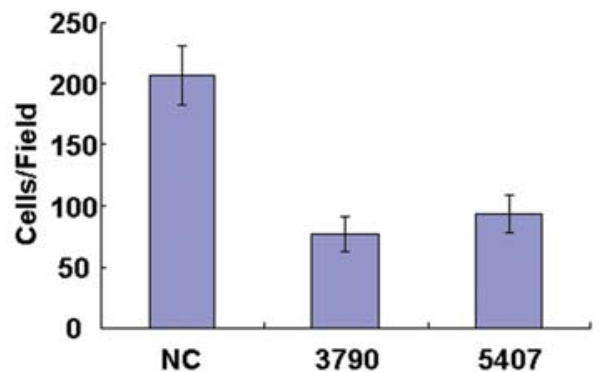
Figure 2. Knockdown of Notch-4 inhibiting the invasion of ACC-M cells. (A) Western blot results of the knockdown of Notch-4 expression in ACC-M cells
by siRNAs. (B) Representative photomicrographs of the results of cell invasion assays in ACC-M cells. Cell invasion through transwell chambers with Matrigel was measured by counting the trespassed cells. Counts of the trespassed cells were presented as the mean values per field from at least five randomly selected low-powered fields (x200) from three independent experiments (C) (error bars, mean \pm SD).

Table II. The expression of Notch-4 in the tissues of adenoid cystic carcinoma with or without metastasis and recurrence cases.

\begin{tabular}{lccccc}
\hline Group & Cases & Negative & Positive & Strong positive & P-value ${ }^{\mathrm{a}}$ \\
\hline Metastasis or recurrence & 23 & 7 & 6 & 10 & 0.017 \\
Non-metastasis & 57 & 31 & 16 & 10 & \\
\hline
\end{tabular}

aRank-sum test $\mathrm{Z}=-2.378 ; \mathrm{p}<0.05$.

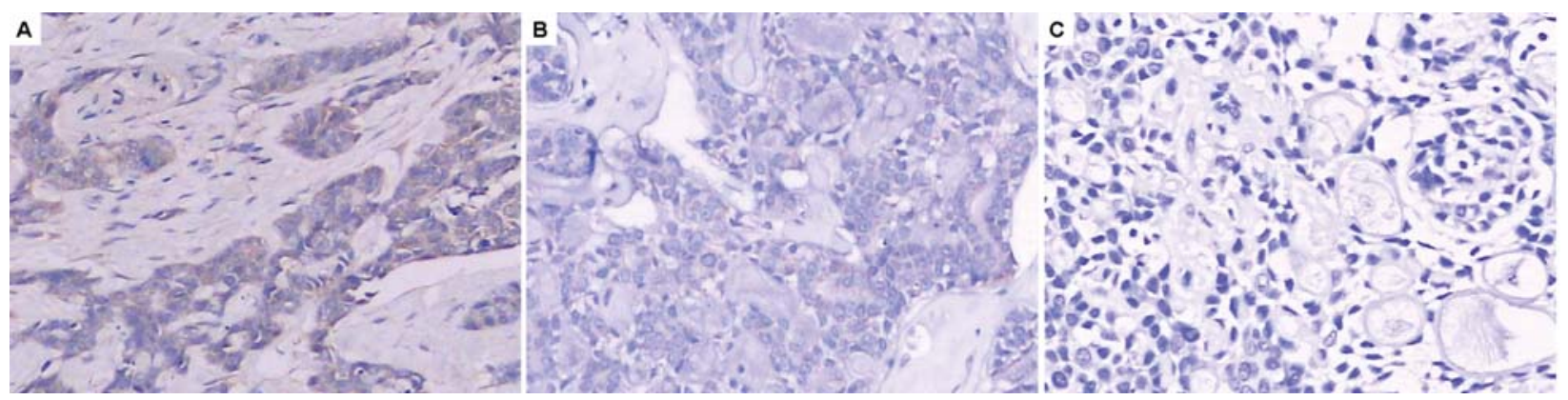

Figure 3. Expression analysis of Notch-4 protein in SACC tissues by immunohistochemistry (x400). (A) The moderate immunopositivity of Notch-4 in the SACC cancer cells; (B) the low immunopositivity of Notch-4 in the SACC cancer cells; (C) negative expression of Notch-4 in the SACC tissues without metastasis and recurrence.

ACC-M cells (Fig. 2B and C, p<0.05, n=3). However, the knockdown of Notch-1 and Notch-2 did not have the same effect (data not shown). These results suggest that Notch-4 upregulation may contribute to the highly metastatic ability of ACC-M cells.
Notch-4 is overexpressed in clinical metastatic tissue samples. To further explore the role of Notch-4 in the metastasis and progression of adenoid cystic carcinoma, Notch-4 expression in the tissue samples of adenoid cystic carcinoma were examined by immunohistochemistry. Of 
these samples, 23 cases had metastasis and recurrence, and the other 57 cases without metastasis and recurrence served as control. As shown in Table II and Fig. 3, Notch-4 expression levels were significantly higher in the tissues with metastasis and recurrence compared to those of control samples $(\mathrm{p}<0.05)$. This result indicates that Notch-4 may play an important role in the metastasis of adenoid cystic carcinoma.

\section{Discussion}

Metastasis is the final and most life-threatening stage of cancer progression. It causes $90 \%$ of human cancer death (12). Therefore, it is essential to define the metastatic mechanisms for improving patient survival. In SACC patients, lung metastasis is the major cause of death. Non-metastatic SACC usually can be effectively treated by surgery and chemotherapy, and the survival rate is higher than that of metastatic SACC. Thus, a better understanding of the mechanisms regulating SACC invasion and metastasis is likely to help improving the overall survival of SACC patients.

The Notch signaling pathway is a highly conserved cellsignaling cascade present in most multicellular organisms. It is important for cell-cell communication and involves gene regulation mechanisms that control multiple cell differentiation processes during embryonic and adult life. Mammals possess four different Notch receptors: Notch-1, Notch-2, Notch-3, and Notch-4.

According to the literature, Notch family members are related to many types of cancer, such as $\mathrm{T}$ cell leukemia (13), mammary tumors (14), pancreatic cancer (15), and so on. Notch-1 is considered as a p53 target gene, involved in human keratinocyte tumors (16). In oral squamous cell carcinoma, Notch-4 deregulation is implied to be related to the deregulation of developmental and differentiation pathways, as well as cell fate misspecification (17). Notch-4 also seems to be involved in the proliferation of human endometrium (18). The changes of Notch-4 expression level may induce the expression of two pro-angiogenic genes, NF-кB and MMP2, in cerebral endothelial cells (19). MMP2 may be associated with the development and metastasis of adenoid cystic carcinoma (4). Delbosc et al also demonstrated that MMP activity reduction occurs through the modulation of Notch signaling (20). Therefore, it is possible that Notch-4 plays a role in cancer progression by regulating MMP2 expression.

To assess the expression pattern of the Notch family members in SACC, we examined the expression of Notch family members in ACC-M and ACC-2 by real-time PCR. The results showed that Notch-1, Notch-2, and Notch-4 were upregulated in ACC-M compared to their expression in ACC-2. The difference of Notch-3 expression between two cell lines was not statistically significant. ACC-M has a higher metastatic ability than ACC-2. Thus, the expression of Notch-1, Notch-2, and Notch-4 may affect the metastasis of SACC cell lines.

Previous studies showed that Notch family members could affect the invasion and metastasis of certain tumors. It was showed that vascular endothelial growth factor and
Notch-1 expression are significantly related to the cervical lymph node metastasis and the depth of invasion in tongue cancer patients (21). The small interfering RNA-mediated knockdown of Notch-1 in prostate cancer cells dramatically decreased their invasion (22). Some previous studies indicated that the Notch pathway has a function in regulating osteosarcoma metastasis, because the inhibition of Notch/HES1 signaling can suppress osteosarcoma metastasis in vivo (23). Reinders et al found that Notch-4 RNA expression was decreased in tumor tissues of head and neck squamous cell carcinoma (24). Although previous research showed that Notch-4 could subvert normal epithelial morphogenesis and promote the invasion of the extracellular matrix (25), in our study, the knockdown of Notch-1 and Notch-2 did not disrupt their expression in ACC-M cells. However, both siRNAs targeting Notch-4 efficiently reduced Notch-4 expression in ACC-M cells, and they significantly inhibited the invasion of ACC-M cells. We concluded that Notch-1 and Notch-2 may be expressed in SACC, but have little effect on SACC metastasis. Notch-4 may play a key role in SACC metastasis, and it can reduce the metastatic ability of ACC-M.

However, contrary results have been reported in terms of the exact function of Notch family in cancer progression. Nam et al reported that the overexpression of Notch-1 and its ligands (DLL-1 and Jag-1) is critical for the survival and proliferation of glioma cells, and the inactivation of Notch signaling can significantly inhibit the migration and invasion of Br4 cells (26). Leong et al observed that Notch-4 activation in human dermal microvascular endothelial cells inhibited endothelial cell sprouting and the vascular endothelial growth factor-induced angiogenesis in the chick chorioallantoic membrane in vivo (27). However, some studies also showed that in human renal cancer cell lines, the Notch-4 expression level was markedly downregulated. Moreover, a previous study showed that Notch-4 expression was absent or significantly decreased in renal cell carcinoma tissues compared to the adjacent non-neoplastic tissues (28). Some researchers found that Notch-4 protein was expressed in both cytoplasm and nucleus of hepatocellular carcinoma (HCC) cells, and compared to the adjacent non-tumor tissue samples, its expression was upregulated in HCC tissue samples (29). By immunohistochemistry assay, we found that Notch-4 was frequently expressed in most of the tissues with metastasis and recurrence, and its expression was significantly higher in the SACC tissues with metastasis and recurrence compared to that of the non-metastatic control tissues. Therefore, we concluded that Notch- 4 upregulation contributes to the highly metastatic ability of SACC.

Gene therapy is the emerging modality of cancer treatment. It is therefore important to define the crucial genes involved in cancer. So far, many genes have been linked to SACC. Hu et al demonstrated that the lung metastasis of SACC might be related to epiregulin (30). Cyclin D1 and cortactin were considered to contribute to SACC metastasis (31). MMP2, MMP7, MMP9, and MMP15 may also be related to SACC metastasis (4).

In our study, we revealed that among all four Notch family members, Notch-4 activation might contribute to SACC metastasis, although it may not be the only critical 
factor. Therefore, our results suggest that Notch-4 inhibitors may have potential therapeutic application in treating metastatic SACC patients. Further investigations are warranted to more precisely determine the roles of Notch-4 in SACC.

\section{Acknowledgements}

This project was supported by the Natural Science Foundation of Fujian Province of China (No. C0710011) and the Key Project of Science and Technology Foundation of Fujian Province of China (No. 2008Y0041).

\section{References}

1. Lima SS, Soares AF, Amorim RF and Freitas Rde A: Epidemiologic profile of salivary gland neoplasms: analysis of 245 cases. Rev Bras Otorinolaryngol 71: 335-340, 2005.

2. Sherigar JM, Finnegan J, McManus D, Lioe TF and Spence RA: Extra salivary adenoid cystic carcinoma; report of two cases. Ulster Med J 75: 223-225, 2006.

3. Umeda M, Nishimatsu N, Masago H, Ishida Y, Yokoo S, Fujioka M, Shibuya Y and Komori T: Tumor-doubling time and onset of pulmonary metastasis from adenoid cystic carcinoma of the salivary gland. Oral Surg Oral Med Oral Pathol Oral Radiol Endod 88: 473-478, 1999.

4. Lu YG, Zhou HY, Ding LC, Mei Y, Xiong RH, Deng SS and Yang HJ: Analysis of differential expression genes related to different metastasis potential of adenoid cystic carcinoma using restriction fragments differential display PCR. Zhonghua Yi Xue Yi Chuan Xue Za Zhi 23: 505-510, 2006.

5. Zhou CX and Gao Y: Aberrant expression of beta-catenin, Pin1 and cyclin D1 in salivary adenoid cystic carcinoma: relation to tumor proliferation and metastasis. Oncol Rep 16: 505-511, 2006.

6. Go MJ, Eastman DS and Artavanis-Tsakonas S: Cell proliferation control by Notch signaling in Drosophila development. Development 125: 2031-2040, 1998.

7. Shelly LL, Fuchs C and Miele L: Notch-1 inhibits apoptosis in murine erythroleukemia cells and is necessary for differentiation induced by hybrid polar compounds. J Cell Biochem 73: 164-175, 1999.

8. Allenspach EJ, Aster JC and Pear WS: Notch signaling in cancer. Cancer Biol Ther 1: 466-476, 2002.

9. GP Dotto: Notch tumor suppressor function. Oncogene 27: 5115-5123, 2008.

10. Leethanakul C, Patel V, Gillespie J, Pallente M, Ensley JF, Koontongkaew S, Liotta LA, Emmert-Buck M and Gutkind JS: Distinct pattern of expression of differentiation and growthrelated genes in squamous cell carcinomas of the head and neck revealed by the use of laser capture microdissection and cDNA arrays. Oncogene 19: 3220-3224, 2000.

11. Livak KJ and Schmittgen TD: Analysis of relative gene expression data using real-time quantitative PCR and the 2(-Delta Delta C(T)) method. Methods 25: 402-408, 2001.

12. Sporn MB: The war on cancer. Lancet 347: 1377-1381, 1996.

13. Chadwick N, Zeef L, Portillo V, Fennessy C, Warrander F, Hoyle S and Buckle AM: Identification of novel Notch target genes in T cell leukaemia. Mol Cancer 8: 35, 2009.

14. Hu C, Diévart A, Lupien M, Calvo E, Tremblay G and Jolicoeur P: Overexpression of activated murine Notch1 and Notch3 in transgenic mice blocks mammary gland development and induces mammary tumors. Am J Pathol 168: 973-990, 2006.

15. Mysliwiec $P$ and Boucher MJ: Targeting Notch signaling in pancreatic cancer patients, rationale for new therapy. Adv Med Sci 54: 1-7, 2009.
16. Lefort K, Mandinova A, Ostano P, Kolev V, Calpini V, Kolfschoten I, Devgan V, Lieb J, Raffoul W, Hohl D, Neel V, Garlick J, Chiorino G and Dotto GP: Notch1 is a p53 target gene involved in human keratinocyte tumor suppression through negative regulation of ROCK1/2 and MRCKalpha kinases. Genes Dev 21: 562-577, 2007.

17. Snijders AM, Schmidt BL, Fridlyand J, Dekker N, Pinkel D, Jordan RC and Albertson DG: Rare amplicons implicate frequent deregulation of cell fate specification pathways in oral squamous cell carcinoma. Oncogene 24: 4232-4242, 2005.

18. Cobellis L, Caprio F, Trabucco E, Mastrogiacomo A, Coppola G, Manente L, Colacurci N, De Falco M and De Luca A: The pattern of expression of Notch protein members in normal and pathological endometrium. J Anat 213: 464-472, 2008.

19. Grigorian A, Hurford R, Chao Y, Patrick C and Langford TD: Alterations in the Notch4 pathway in cerebral endothelial cells by the HIV aspartyl protease inhibitor, nelfinavir. BMC Neurosci 9: 27, 2008.

20. Delbosc S, Glorian M, Le Port AS, Béréziat G, Andréani M and Limon I: The benefit of docosahexanoic acid on the migration of vascular smooth muscle cells is partially dependent on Notch regulation of MMP-2/-9. Am J Pathol 172: 1430-1440, 2008.

21. Joo YH, Jung CK, Kim MS and Sun DI: Relationship between vascular endothelial growth factor and Notch1 expression and lymphatic metastasis in tongue cancer. Otolaryngol Head Neck Surg 140: 512-518, 2009.

22. Bin Hafeez B, Adhami VM, Asim M, Siddiqui IA, Bhat KM, Zhong W, Saleem M, Din M, Setaluri V and Mukhtar H: Targeted knockdown of Notch1 inhibits invasion of human prostate cancer cells concomitant with inhibition of matrix metalloproteinase-9 and urokinase plasminogen activator. Clin Cancer Res 15: 452-459, 2009.

23. Zhang P, Yang Y, Zweidler-McKay PA and Hughes DP: Critical role of notch signaling in osteosarcoma invasion and metastasis. Clin Cancer Res 14: 2962-2969, 2008.

24. Reinders J, Rozemuller EH, van der Weide P, Oka A, Slootweg PJ, Inoko $\mathrm{H}$ and Tilanus MG: Genes in the HLA region indicative for head and neck squamous cell carcinoma. Mol Immunol 44: 848-855, 2007.

25. Soriano JV, Uyttendaele H, Kitajewski J and Montesano R: Expression of an activated Notch4(int-3) oncoprotein disrupts morphogenesis and induces an invasive phenotype in mammary epithelial cells in vitro. Int J Cancer 86: 652-659, 2000.

26. Nam DH, Jeon HM, Kim S, Kim MH, Lee YJ, Lee MS, Kim H, Joo KM, Lee DS, Price JE, Bang SI and Park WY: Activation of notch signaling in a xenograft model of brain metastasis. Clin Cancer Res 14: 4059-4066, 2008.

27. Leong KG, Hu X, Li L, Noseda M, Larrivée B, Hull C, Hood L, Wong $\mathrm{F}$ and Karsan A: Activated Notch4 inhibits angiogenesis: role of beta 1-integrin activation. Mol Cell Biol 22: 2830-2841, 2002.

28. Sun S, Du R, Gao J, Ning X, Xie H, Lin X, Liu J and Fan D: Expression and clinical significance of Notch receptors in human renal cell carcinoma. Pathology 41: 335-341, 2009.

29. Gao J, Song Z, Chen Y, Xia L, Wang J, Fan R, Du R, Zhang F, Hong L, Song J, Zou X, Xu H, Zheng G, Liu J and Fan D: Deregulated expression of Notch receptors in human hepatocellular carcinoma. Dig Liver Dis 40: 114-121, 2008.

30. Hu K, Li SL, Gan YH, Wang CY and Yu GY: Epiregulin promotes migration and invasion of salivary adenoid cystic carcinoma cell line SACC-83 through activation of ERK and Akt. Oral Oncol 45: 156-163, 2009.

31. Greer RO Jr, Said S, Shroyer KR, Marileila VG and Weed SA: Overexpression of cyclin D1 and cortactin is primarily independent of gene amplification in salivary gland adenoid cystic carcinoma. Oral Oncol 43: 735-741, 2007. 